\title{
Computational Modeling of Driver Distraction by Integrating Cognitive and Agent-based Traffic Simulation Models
}

\author{
Seyed Hossein Hosseini Nourzad ${ }^{1}$, Dario D. Salvucci ${ }^{2}$ and Anu Pradhan ${ }^{3}$ \\ ${ }^{1} \mathrm{PhD}$ Candidate, Department of Civil, Architectural and Environmental Engineering, \\ Drexel University, Philadelphia, PA 19104: PH (215) 429-0700; FAX (215) 895- \\ 1363; email: hnourzad@drexel.edu \\ ${ }^{2}$ Professor of Computer Science, College of Computing \& Informatics, Drexel \\ University, Philadelphia, PA 19104: PH (215) 895-2674; FAX (215) 895-0545; \\ email: salvucci@drexel.edu \\ ${ }^{3}$ Assistant Professor, Department of Civil, Architectural and Environmental \\ Engineering, Drexel University, Philadelphia, PA 19104; PH (215) 571-3540; FAX \\ (215) 895-1363; email: pradhan@drexel.edu
}

\begin{abstract}
The U.S. National Highway Traffic Safety Administration has reported that the major cause of vehicle crashes in the United States has been driver inattention and distraction. Among various types of distraction, cell-phone dialing and text messaging have been shown to significantly degrade driving performance. Existing studies have mainly focused on driver distraction scenarios with one or a few vehicles, and have generally not looked at large-scale simulation of many vehicles. In contrast, currently available software for simulating traffic with many vehicles (e.g., CORSIM and VISSIM) has generally not considered the effects of driver distraction on driver/vehicle behavior. To overcome these limitations, we propose a computational modeling framework that integrates a cognitive model of distraction (i.e., Distract-R) and an agent-based traffic (micro-) simulation model (i.e., VISSIM). The framework aims to enable transportation modelers to easily set up virtual experiments and evaluate the impact of distracted drivers on large-scale networks. We validate the framework using two existing experimental data sets. Preliminary results indicate that the framework can account for statistically significant changes in speed fluctuation and headway distance in the presence of a significant number of distracted drivers.
\end{abstract}

\section{INTRODUCTION}

Networked infrastructures, such as transportation networks and telecommunication systems, are of utmost importance to our modern societies. Moreover, interconnection among these infrastructures has many benefits. For instance, current mapping systems on cell-phones improve overall traffic conditions on road networks by guiding individual drivers to find their optimal routes. Despite many advantages of telecommunication systems, misuse of such systems might threaten vulnerability of transportation networks. For example, cell-phone use while driving degrades driver performance and, as a result, negatively impacts traffic 
conditions on a road network. According to the U.S. National Highway Traffic Safety Administration, driver distraction is a major cause of vehicle crashes in the United States (Young, et al., 2007). Among the various types of distraction, cell-phone dialing and text messaging have been shown to significantly degrade driving performance (Salvucci, 2013, Stavrinos, et al., 2013). Therefore, it is necessary to consider large-scale effects of distraction to analyze vulnerability of a road network.

Currently, there exists a gap in large-scale study and distraction simulation. Existing works (i.e., naturalistic experiments, laboratory experiments, statistical studies and computational modeling) have primarily focused on driver distraction scenarios with either one or a few vehicles (Box, 2009, Salvucci, 2013, Stavrinos, et al., 2013, Young, et al., 2007). In contrast, available software for simulating traffic with many vehicles (e.g., CORSIM and VISSIM) has not generally considered the effects of driver distraction. Therefore, it is necessary to develop a framework to assess large-scale effects of distraction, especially if several drivers in a network are distracted at a given time. For example, if 5\% of drivers on road use cell-phones, how will it affect traffic flow dynamics? Or in the case of an emergency, what are the impacts of dramatic changes in cell-phone use? In this paper, we formalize a computational framework that integrates a cognitive model of distraction (i.e., Distract-R) and an agent-based traffic (micro-) simulation model (i.e., VISSIM). The framework is then validated using two existing experiments. The framework is applied to analyze the effects of text messaging on a real traffic model for the Philadelphia metropolitan area.

\section{BACKGROUND RESEARCH}

Distraction has been typically categorized into four types: (1) visual, (2) auditory, (3) physical, and (4) cognitive distractions (Young, et al., 2007). There are many debates regarding the forms of devices that will lead to the greatest degradation in driving performance (Salvucci, 2009). In general, driving is primarily a visualmanual task (Young, et al., 2007). Therefore, according to Wickens' multiple resource theory, any device that requires visual and/or manual responses should cause greater degradation in driving performance (Wickens, 2002). For instance, researchers at Virginia Tech Transportation Institute (VTTI) showed that textmessaging, which is a manual manipulation of cell-phones, is associated with 23 times the risk factor compared to normal driving (Box, 2009).

During the last decade, driver distraction due to performing secondary tasks has received a great deal of attention (Cooper, et al., 2009, Fitch, et al., 2013, Salvucci, 2009, Wilson and Stimpson, 2010, Young, et al., 2007). These studies can be categorized into four groups. The first group includes naturalistic experiments that record drivers' behavior using cameras and kinematic sensors in real-world driving conditions (Box, 2009, Charlton, et al., 2013, Fitch, et al., 2013). While these experiments are more realistic and may capture the actual driving situations better than other three groups of studies, they are expensive, time-consuming, and risky. The second group is the laboratory simulations, which are the most common methods to investigate driver distraction (Beede and Kass, 2006, Cooper, et al., 2009, Strayer, et al., 2006, Young, et al., 2007). Laboratory experiments are less dangerous 
compared to the naturalistic experiments, but they are still time-consuming and expensive (Salvucci, 2009). The third group includes population-based studies, which use existing databases (e.g., Fatality Analysis Reporting System database) to discover the interrelation between crash trend and secondary tasks (e.g., text-messaging) (Redelmeier and Tibshirani, 1997, Wilson and Stimpson, 2010). However, the accuracy of these studies depends on completeness of employed databases. To overcome the limitations of the abovementioned three groups, the researchers analyzed the use of cognitive models in predicting distraction (Salvucci, 2009, Salvucci, 2013). For example, the Distract-R uses cognitive models as an internal engine to prototype and evaluate the use of devices in vehicle (Salvucci, 2009). The main benefit of the fourth group (i.e., computational experiments) is that they are fast, easy to set up and repeatable. For instance, the Distract-R predicts driver performance 1000 times faster than real time. However, they need validation to ensure that their predictions correspond to real-world measures and values.

Existing studies have mainly focused on driver distraction scenarios with either one or a few vehicles, and have generally not looked at large-scale simulation of many vehicles. A few network-wide attempts were performed to assess the impacts of driver distraction on the surrounding traffic (Cooper, et al., 2009, Salvucci, 2013, Stavrinos, et al., 2013). For instance, Stavrinos et al (2013) used laboratory simulation to analyze behavior of young adults while engaged in cell-phone conversation and text messaging in different driving conditions (i.e., free flow, stable flow, and oversaturation). They concluded that distraction (especially text messaging) negatively impacted the traffic by greater speed deviations, fewer numbers of lane changes, reduced safety, and more lane deviations (Stavrinos, et al., 2013). In another recent study, Salvucci (2013) developed a computational cognitive model based on the Distract-R to predict the effects of driver distraction (e.g., cell-phone dialing) on other vehicles around distracted driver's vehicle. He simulated two distinct carfollowing scenarios in which 16 drivers drove (a) on a straight single-lane roadway, and (b) in a circular loop of traffic. This is the first study which assessed simultaneous impacts of multiple (e.g., 3 out of 16) distracted drivers on traffic conditions.

While existing studies investigated the impacts of distraction at driver-level, they have some limitations. First, the impacts of simultaneous distraction of significant number of drivers have not yet investigated. Second, the evaluation of network-level impacts of distraction needs large-scale experiments, which are not easy to set up using physical simulators. Third, real scenarios representing different combinations of distraction types are challenging to define using the existing methods. For instance, currently it is not easily possible to evaluate the impacts of distraction on a large network in which $5 \%$ of drivers use their cell-phone for conversation and $8 \%$ of drivers use their cell-phone for text messaging.

\section{RESEARCH APPROACH}

The overall proposed framework to model vulnerability of a road network under driver distraction is presented in Figure 1. The framework has two phases: (1) distraction simulation and (2) integration of distraction and traffic simulation models. 
In the first phase, for each distraction type (e.g., text messaging), a distraction time profile will be estimated using a cognitive model (i.e., Distract-R). The distraction time profile is a time profile which shows the status of a driver in each time step whether he is distracted or not (e.g., see Figure 3-c). To generate the distraction time profile, the Distract-R model takes device prototype, driver behavior and driving environment as inputs. After cognitive modeling, the Distract-R returns the distraction time profile as an output. The outputs of the first phase for different distraction types are stored in the form of a database that could be used by the traffic simulation model during a period of distraction.

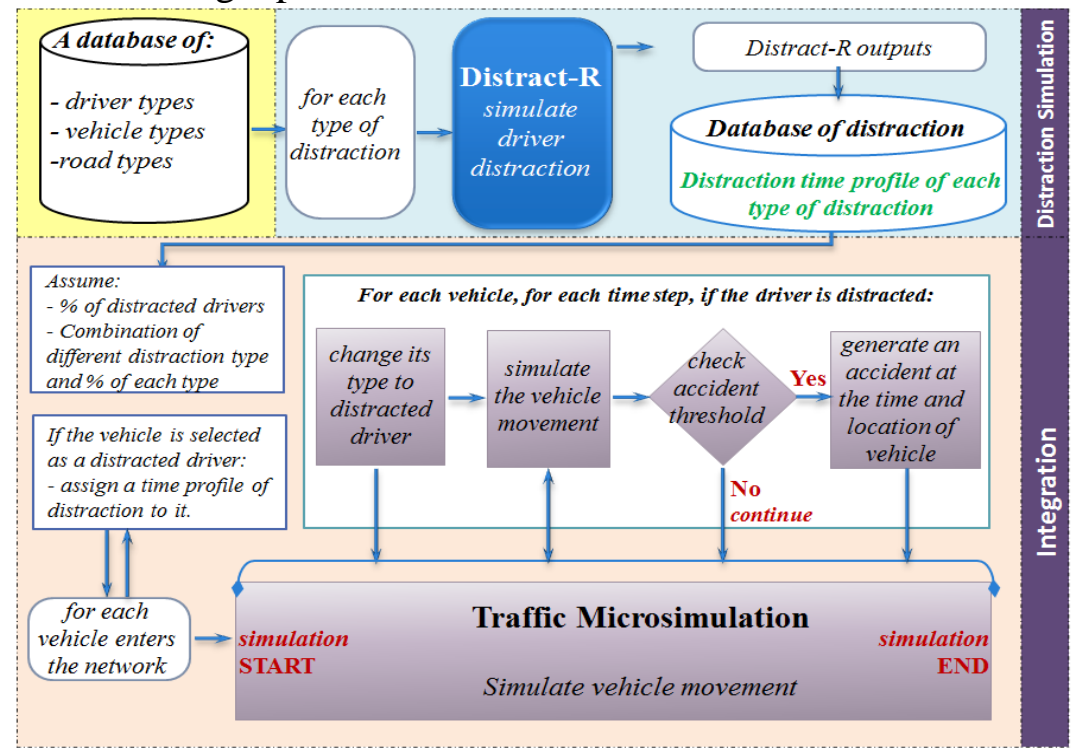

Figure 1. Research approach for integrating distraction and traffic simulation models

In the second phase, the distraction time profiles are integrated into a largescale traffic simulation using a microscopic simulation model (i.e., VISSIM). We utilize a microscopic simulation model because we need to know the precise position of each vehicle at each time step as small as one tenth of a second. During the simulation, for each vehicle, the driver behavior parameters (e.g., car-following parameters) are adjusted whenever the driver is distracted according to distraction time profile. To do so, we selected VISSIM simulation model which enables us to customize vehicle properties through COM (Component Object Model) functionality with Python programming environment. When a vehicle enters the network, if it is selected as distracted driver, a distraction time profile is assigned to the vehicle. Movements of all vehicles are simulated at each time step according to their distraction status until they reach their destinations. This way, we integrate distraction effects into the large-scale traffic simulation. The framework enables us to test various percentages of distracted drivers and various combinations of distraction types.

Finally, since the proposed framework is based on computational simulation, we should validate this approach by replicating existing experiments, such as (Salvucci, 2013). It is a recent study and the only one which assessed the impacts of a few distracted drivers on traffic conditions. We attempt to replicate the experiments to evaluate if we observe the same change in traffic conditions. Furthermore, there is 
a recent naturalistic report published by the U.S. National Highway Traffic Safety Administration, which reported overall naturalistic results of distraction (e.g., speed fluctuation) in the case of phone conversation and text messaging (Fitch, et al., 2013). We compare our results with these naturalistic results as well.

\section{RESULTS}

In this section, in addition to validation results (Figure 2), we present preliminary results for large-scale simulating of multiple distracted drivers in a real road network in the Philadelphia area (Figure 3).

Validation results. We set up two experiments similar to those performed by Salvucci (2013). Figure 2 shows configurations and results of the two experiments, in which sixteen drivers drove on a straight roadway (Figure 2-a) and in a circular loop (Figure 2-b). In this case, the researcher studied distraction due to cell-phone dialing. For more details of the experiments, interested readers can refer to (Salvucci, 2013).

(a) Standard Car-Following Setup:

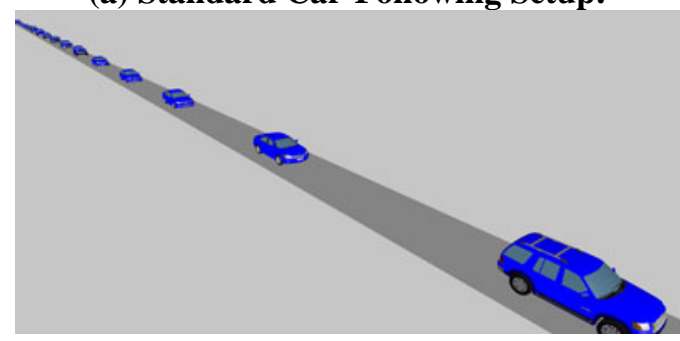

(b) Circular Car-Following Setup:

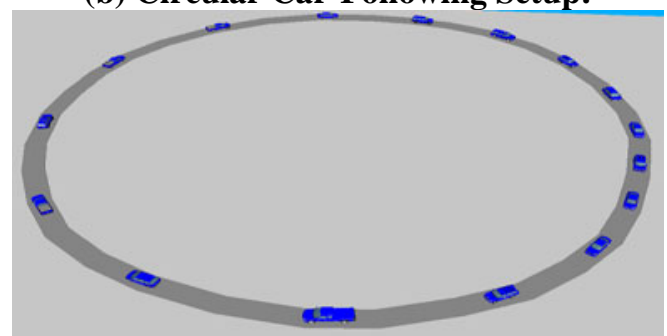

(c) Standard Car-Following Results:

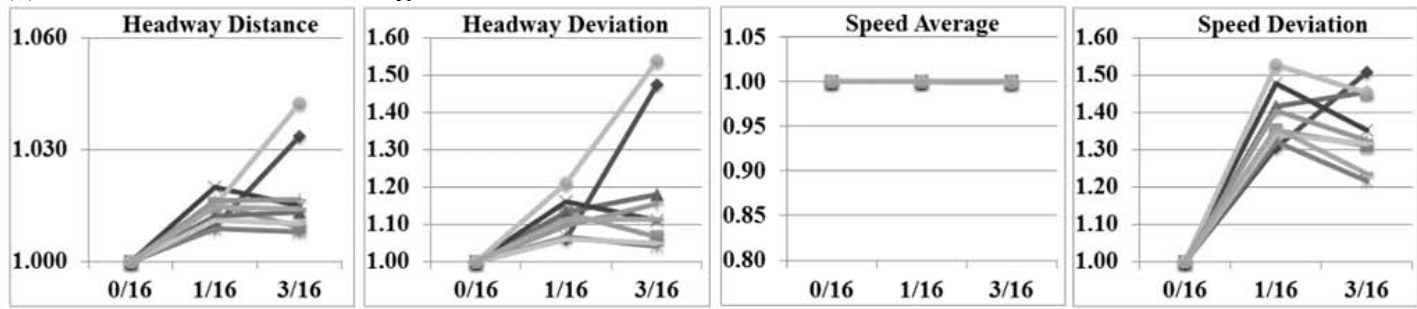

(d) Circular Car-Following Results:

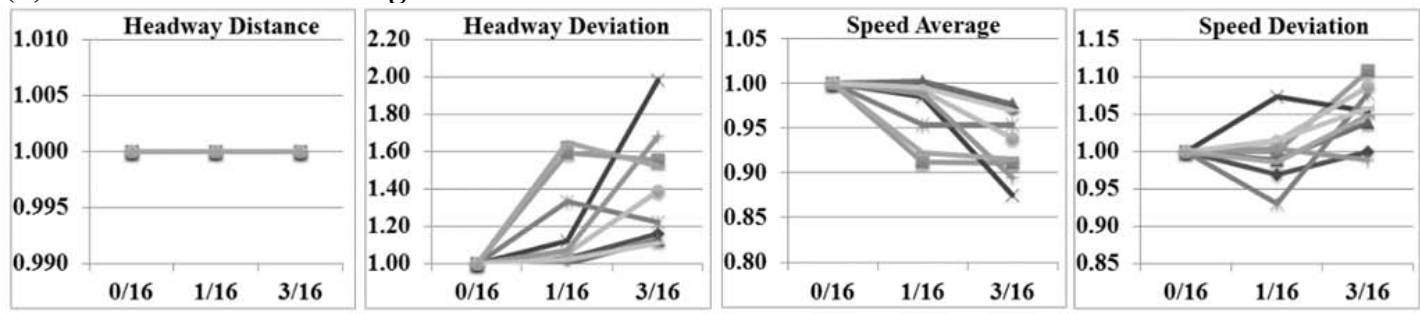

Figure 2. Validation case studies. Each graph line represents a group of model drivers simulated across three conditions, namely with 0,1 , or 3 distracted drivers (performing the dialing task) out of 16 total drivers. For each graph line, the results are divided by the value of $0 / 16$. For example, 1.60 for Headway Deviation means $60 \%$ increase compared to no-distraction case.

Salvucci (2013) concluded that for a standard car-following scenario, headway distance average increased a small amount, headway deviation did not significantly vary, speed average decreased for some groups, and speed deviation increased. These 
results were observed here (see Figure 2-c), except for headway deviation which increased in our test. For a circular car-following scenario, Salvucci (2013) reported that headway distance average remained constant, headway deviation grew slightly, speed average decreased, and speed deviation increased. All these results were observed in our test (see Figure 2-d). Moreover, the increase in speed deviation in both of the test cases is in line with the results of VTTI naturalistic experiment (Fitch, et al., 2013).

\section{Preliminary results of a large-scale distraction simulation}

The proposed framework enables transportation modelers to easily set up virtual experiments and evaluate the impact of distracted drivers on traffic conditions of large-scale networks. As a preliminary example, we employed the proposed framework for a real case study from the Philadelphia area. The model was built around the interchange of Interstates 476 and 76 including about one mile of each Interstate (Figure 3-a). We simulated traffic across three conditions, where (i) $0 \%$, (ii) $10 \%$ and (iii) $20 \%$ of drivers were distracted by text messaging. First, we generated the time profile of distraction due to text-messaging using Distract-R (Figure 3-c). The generated time profile is in line with VTTI naturalistic results, in which they reported that on average text messaging lasted 36.4 s (Fitch, et al., 2013). Then, we repeated three 10-minute-long traffic simulations during PM hours with varying percentages of distracted drivers.

(a) I-76/I-476 Interchange Model:

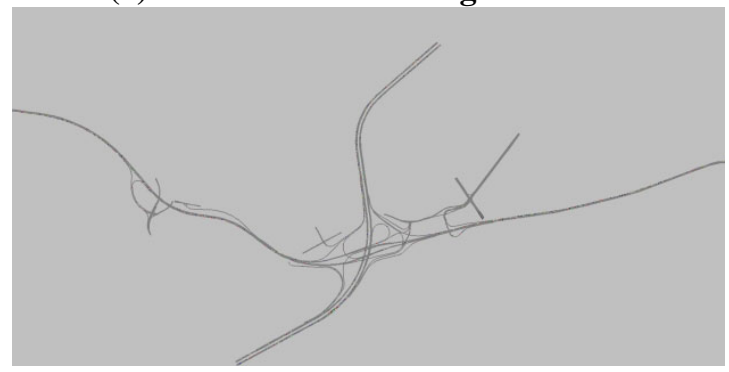

(b) A Sample 3D Snapshot from the Model:

(c) Snapshot from Distraction Time Profile:

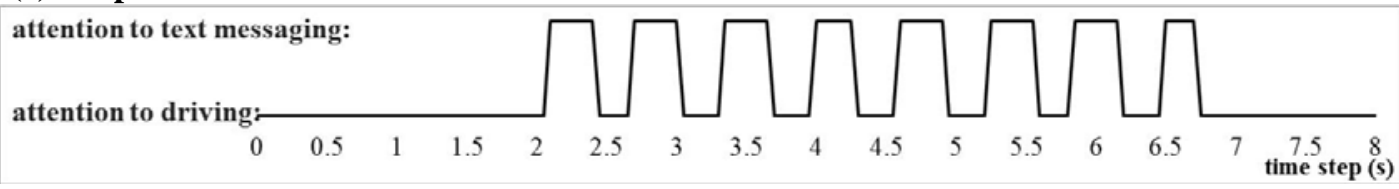

(d) The Results of Large-scale Distraction Simulation:

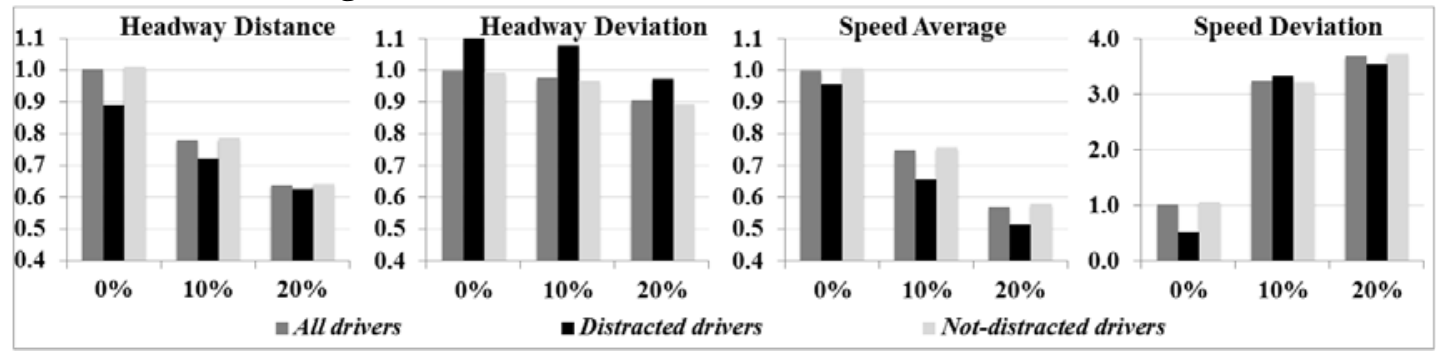

Figure 3. Sample visualizations and preliminary results. The results show the impact of different distraction levels (namely $0 \%, 10 \%$ and $20 \%$ of drivers use cell-phone for text messaging) on traffic conditions (i.e., headway distance and speed). 
The preliminary results show that when the number of distracted drivers increases, on average, drivers decrease their headway distance and speed. However, speed deviation increases significantly. In addition to the overall results for all drivers, Figure 3-d plots the results for distracted and not-distracted drivers, separately. Although the trends of change (either increasing or decreasing) are similar for different driver categories (i.e., all, distracted, and not-distracted), distraction affects the distracted drivers more than the surrounding vehicles. However, the overall impacts of distraction may be considered significant in the sense that a sizeable proportion of vehicles are driving in an impaired capacity.

\section{DISCUSSION}

Recent research has explored how traffic efficiency may be reduced by different factors, such as presence of slow vehicles and greater speed variability (Cooper, et al., 2009). Our preliminary results indicate that, on average, distracted drivers proceed slower than surrounding traffic and increase speed variability. Therefore, they reduce traffic efficiency. In addition, speed variability in a network translates to traffic safety because deviation in speed increases the need for responding to suddenonset events and thus increases the risk of crash. Thus, our results may indicate a drop in traffic safety in the presence of many distracted drivers.

There are many debates regarding the change in headway distance due to distraction. Some researchers concluded that the headway distance increased (Strayer, et al., 2006), while others reported a decrease in this measure (Rosenbloom, 2006). Our preliminary results indicate a significant decrease in headway distance, which again might translate to decrease in traffic safety. In addition, although we expected to see an increasing trend in headway deviation (by increasing distraction percentage), our results show a decrease in headway deviation which seems to contradict with our preliminary assumption. However, we may consider coefficient of variation of headway distance which is defined as the proportion of headway deviation to average headway distance. The coefficient of variation of headway increases with the increase of distraction level. For example, headway coefficient of variation is $0.76,0.93$, and 0.96 for $0 \%, 10 \%$ and $20 \%$ distraction, respectively. Generally speaking, we conclude that distraction increases the variability in traffic conditions and as a results decreases safety and efficiency of traffic.

\section{CONCLUSION}

The present study is an initial step for evaluating large-scale impacts of realworld distraction scenarios. In reality, a significant number of drivers may be distracted by various types (e.g., dialing, text messaging, and phone conversation) while driving at any given time. To develop an approach for simulating such a real scenario, the proposed framework integrates a cognitive model of distraction (i.e., Distract-R) and an agent-based traffic (micro-) simulation model (i.e., VISSIM). However, no study is without limitations and our study is no different. For example, we modeled driving behavior in a computational environment which is based on validated simulation models for evaluating large-scale impacts of distraction. Future work might consider how to combine large-scale simulations with larger-scale 
experiments (naturalistic or otherwise) to assess whether such inefficiencies translate to real-world scenarios.

\section{ACKNOWLEDGMENT}

The authors would like to thank Mr. Michael Becker and Mr. Keith Hartington (from Delaware Valley Regional Planning Commission) for sharing the I-76/I-476 calibrated VISSIM model. Any opinions, findings, conclusions, or recommendations expressed in this report are those of the authors.

\section{REFERENCES}

Beede, K. E., and Kass, S. J. (2006). "Engrossed in Conversation: The Impact of Cell Phones on Simulated Driving Performance." Accident Analysis \& Prevention, 38(2), 415-421.

Box, S. (2009). "New Data from Vtti Provides Insight into Cell Phone Use and Driving Distraction." Virginia Tech Transportation Institute.

Charlton, J. L., Catchlove, M., Scully, M., Koppel, S., and Newstead, S. (2013). "Older Driver Distraction: A Naturalistic Study of Behaviour at Intersections." Accident Analysis \& Prevention.

Cooper, J. M., Vladisavljevic, I., Medeiros-Ward, N., Martin, P. T., and Strayer, D. L. (2009). "An Investigation of Driver Distraction near the Tipping Point of Traffic Flow Stability." Human Factors: The Journal of the Human Factors and Ergonomics Society, 51(2), 261-268.

Fitch, G. M., Soccolich, S. A., Guo, F., McClafferty, J., Fang, Y., Olson, R. L., Perez, M. A., Hanowski, R. J., Hankey, J. M., and Dingus, T. A. (2013). "The Impact of Hand-Held and Hands-Free Cell Phone Use on Driving Performance and Safety-Critical Event Risk."

Redelmeier, D. A., and Tibshirani, R. J. (1997). "Association between Cellular-Telephone Calls and Motor Vehicle Collisions." New England Journal of Medicine, 336(7), 453458.

Rosenbloom, T. (2006). "Driving Performance While Using Cell Phones: An Observational Study." Journal of Safety Research, 37(2), 207-212.

Salvucci, D. D. (2009). "Rapid Prototyping and Evaluation of in-Vehicle Interfaces." Acm T Comput-Hum Int, 16(2), 9:1-9:33.

Salvucci, D. D. (2013). "Distraction Beyond the Driver: Predicting the Effects of in-Vehicle Interaction on Surrounding Traffic."

Stavrinos, D., Jones, J. L., Garner, A. A., Griffin, R., Franklin, C. A., Ball, D., Welburn, S. C., Ball, K. K., Sisiopiku, V. P., and Fine, P. R. (2013). "Impact of Distracted Driving on Safety and Traffic Flow." Accident Analysis \& Prevention.

Strayer, D. L., Drews, F. A., and Crouch, D. J. (2006). "A Comparison of the Cell Phone Driver and the Drunk Driver." Human factors: The journal of the human factors and ergonomics society, 48(2), 381-391.

Wickens, C. D. (2002). "Multiple Resources and Performance Prediction." Theoretical issues in ergonomics science, 3(2), 159-177.

Wilson, F. A., and Stimpson, J. P. (2010). "Trends in Fatalities from Distracted Driving in the United States, 1999 to 2008." American Journal of Public Health, 100(11), 22132219.

Young, K., Regan, M., and Hammer, M. (2007). "Driver Distraction: A Review of the Literature." Distracted driving. Sydney, NSW: Australasian College of Road Safety, 379405. 